\title{
Gate and magnetic field tunable ultrastrong coupling between a magnetoplasmon and the optical mode of an LC cavity
}

\author{
Gian L. Paravicini-Bagliani, ${ }^{*}$ Giacomo Scalari, ${ }^{\dagger}$ Federico Valmorra, Janine Keller, \\ Curdin Maissen, Mattias Beck, and Jérôme Faist \\ Institute for Quantum Electronics, ETH Zurich, Auguste-Piccard-Hof 1, 8093 Zurich, Switzerland
}

(Received 1 March 2017; revised manuscript received 20 April 2017; published 12 May 2017)

\begin{abstract}
The coupling between the optical mode of an LC cavity and a magnetoplasmon is studied by terahertz transmission spectroscopy. The magnetoplasmons are created by etching a high-mobility two-dimensional electron gas into stripes. As a result, we identify three different regimes, depending on the plasmon frequency relative to the cavity frequency. We find a significant coupling to the cyclotron dispersion even in presence of screening of the electric field by the plasmon.
\end{abstract}

DOI: 10.1103/PhysRevB.95.205304

\section{INTRODUCTION}

In the ultrastrong light-matter coupling regime, energy exchange between a cavity and matter excitation occurs at the rate $\Omega$, a significant fraction $(\geqslant 10 \%)$ of the bare cavity $\omega_{\text {cav }}$ and matter frequencies. The interesting features of this system, predicted theoretically [1], triggered strong experimental work towards its practical realization [2-10]. Subwavelength LC resonators on two-dimensional electron gases (2DEGs) in strong perpendicular magnetic fields have been shown to be a highly flexible platform to study ultrastrong light-matter interaction physics in the $\mathrm{THz}$ [8]. The coupling occurs between the resonators' LC mode $\omega_{\text {cav }}$ and the cyclotron transition $\omega_{c}$ between two Landau levels close to the Fermi energy $[8,11]$. This system made it possible to reach record high normalized light-matter coupling ratios $\Omega / \omega_{\text {cav }}$ approaching $100 \%$ [11].

Besides the cyclotron transition, the magnetoplasmon (MP) resonance appearing in $2 \mathrm{DEG}$ stripes represents another excitation with a significant in-plane electric dipole moment. Since it is both magnetic-field and gate tunable, it represents an attractive matter part to perform an ultrastrong coupling experiment. In this work we present a sample geometry, which makes it possible to couple to both MPs and cyclotron transitions simultaneously. This allows us to spectroscopically study the effects of plasmon screening on the cyclotron excitation. Furthermore, the sample geometry is readily expandable to study quantum Hall transport in the presence of the ultrastrong coupling regime in a future experiment.

Stripes of 2DEG have been studied intensively in the past using transport experiments, as well as optical/electronic transmission or reflection measurements and often in magnetic fields. In optical/microwave transmission experiments, the most prominent feature is the plasmon resonance caused by the collective excitation of the entire electron gas first predicted in 1967 [12]. Experimental observations of plasmons

\footnotetext{
*gianpa@phys.ethz.ch

†scalari@phys.ethz.ch
}

Published by the American Physical Society under the terms of the Creative Commons Attribution 4.0 International license. Further distribution of this work must maintain attribution to the author(s) and the published article's title, journal citation, and DOI. have been made with many different materials and device geometries [13-18] and have received continued interest to this date [19] thanks to new materials such as graphene [20-22] and $\mathrm{GaN}$ [23] and new physics such as relativistic effects [24] and plasmons coupled to cavities $[6,9,25]$, as well as for their potential applications [26-28], to name a few.

To lowest order, the plasmon frequency $\omega_{p}$ in the longwavelength approximation is determined by the width $W$ of the 2DEG stripe, its density $n_{s}$, and effective electron mass $m^{*}$, as well as effective dielectric permittivity $\epsilon$ of the surrounding medium [12],

$$
\omega_{p}=\sqrt{\frac{n_{s} e^{2} \pi}{2 m^{*} \epsilon \epsilon_{0} W}} .
$$

In a perpendicular $B$ field, the resulting MP dispersion is well described by

$$
\omega_{M P}^{2}=\omega_{p}^{2}+\omega_{c}^{2},
$$

where $\omega_{c}=\frac{e B}{m^{*}}$ is the cyclotron dispersion to which the MP dispersion converges in the high-frequency limit. This is often considered as a hybridization of the plasmon with the cyclotron dispersion [16,17,29]. More recent microwave experiments show that these excitations are coexisting [30,31], but the cyclotron transition remains very hard to observe if nearby the MP resonance due to screening of the incident electric field.

We show ultrastrong coupling of MP and cyclotron excitations in the 2DEG stripe to a subwavelength LC cavity. This makes it possible to spectroscopically study their dispersions with a free space optical transmission experiment in the THz. Ultrastrong coupling to MPs has previously been shown in the microwave regime using coplanar microresonators [6] and patch resonators [9] in GaAs-based 2DEGs, as well as using split-ring resonators coupled to graphene nanoribbons [25].

\section{SAMPLE DESIGN AND MEASUREMENT SETUP}

Our samples are based on a $\mathrm{GaAs} / \mathrm{Al}_{0.3} \mathrm{Ga}_{0.7} \mathrm{As}$ single triangular quantum well $90 \mathrm{~nm}$ below the sample surface grown by molecular beam epitaxy. A Si $\delta$-doping layer with density $3.5 \times 10^{12} \mathrm{~cm}^{-2}$ is located $50 \mathrm{~nm}$ below the sample surface. The electron transport mobility is $\mu=2 \times 10^{6} \mathrm{~cm}^{2} / \mathrm{Vs}$ and the electron density is $n_{s}=2.7 \times 10^{11} \mathrm{~cm}^{-2}$ at zero gate 
bias. For samples A1 and A2 we etched several 4-mm-long, $3.4-\mu \mathrm{m}$-wide stripes spaced by $300 \mu \mathrm{m}$. For sample B, the stripes are $1.4 \mu \mathrm{m}$ wide instead. For a gate on sample A2, we add Ohmic contacts by evaporating and annealing $18 / 48 / 15 / 150-n m$-thick $\mathrm{Ge} / \mathrm{Au} / \mathrm{Ni} / \mathrm{Au}$ at both ends of each stripe. A two-dimensional square array of complementary split-ring resonators with an LC resonance frequency of around $500 \mathrm{GHz}$ and a pitch of $300 \mu \mathrm{m}$ is placed on the stripes of all three samples [see Fig. 1(c)]. The patch of 7/200-nm-thick $\mathrm{Ti} / \mathrm{Au}$ into which the complementary resonators are patterned is $3.5 \mathrm{~mm}$ across. To obtain a proper gate covering the entire stripe area of sample A2, a 2-nm-thick chromium layer is deposited on top of the resonators. While this layer gives a conducting layer for a dc gate bias, its thickness is far below the skin depth at a few hundred $\mathrm{GHz}$ and does therefore not inhibit the transmission of $\mathrm{THz}$ light [32], nor does it change the plasmon dispersion [27]. In order to directly measure the bare MP dispersion, we use sample R, which has 3.4- $\mu$ m-wide stripes with a closer spacing of $40 \mu \mathrm{m}$ to have a better spectroscopic signal but still far enough to not change the MP dispersion.

The LC mode of the resonator concentrates most of the electric field across the 4.5- $\mu \mathrm{m}$-wide gap, as shown in the finite element $(\mathrm{FE})$ simulation overlapped as a color map on the scanning electron microscope (SEM) picture of the sample [see Fig. 1(c)]. The color map shows the cavity in-plane electric field distribution $E_{x, y}=\sqrt{\left|E_{x}\right|^{2}+\left|E_{y}\right|^{2}}$, normalized to the incident electric field. It thus shows the local field enhancement factor. See Sec. IV for further details on the FE simulation obtained with Computer Simulation Technology (CST) microwave studio. The effective cavity volume is approximately given by the product of the in-plane area of the slit $4.5 \times 36 \mu \mathrm{m}$ times the out-of-plane extension of the field, which is approximately equal to the gap diameter. Thus, one obtains a cavity volume of $V_{\text {cav }}=3 \times 10^{-5}(\lambda / 2)^{3}$, where $(\lambda / 2)^{3}$ is the free space volume of a photon at $500 \mathrm{GHz}$. This results in strong vacuum electric-field fluctuations on the order of

$$
E_{\mathrm{vac}}=\sqrt{\frac{\hbar \omega_{\mathrm{cav}}}{\epsilon \epsilon_{0} V_{\mathrm{cav}}}} \approx 100 \mathrm{~V} / \mathrm{m}
$$

at the $\mathrm{LC}$ resonance frequency, where $\epsilon=(12.89+1) / 2$.

The field is polarized across the electron gas stripe and hence can couple to cyclotron transitions, requiring an in-plane electric field, as well as to the MP requiring light polarized across the stripe. Therefore, this system is well suited to study the ultrastrong coupling physics of both excitations.

The sample is placed into a helium cryostat with a superconducting magnet in Faraday geometry at a temperature of around $3 \mathrm{~K}$. THz time domain spectroscopy (THz-TDS) is used to perform transmission measurements through the arrays of stripes and resonators (see [11] for more details on the setup).

\section{RESULTS AND DISCUSSION}

Figure 1(a) shows the transmission of sample R normalized to a GaAs substrate measured with THz-TDS for different magnetic fields. The resulting dip in amplitude transmission is around $1 \%$. The black curve shows a fit using the model of the MP dispersion from Eq. (2). A reduced effective stripe
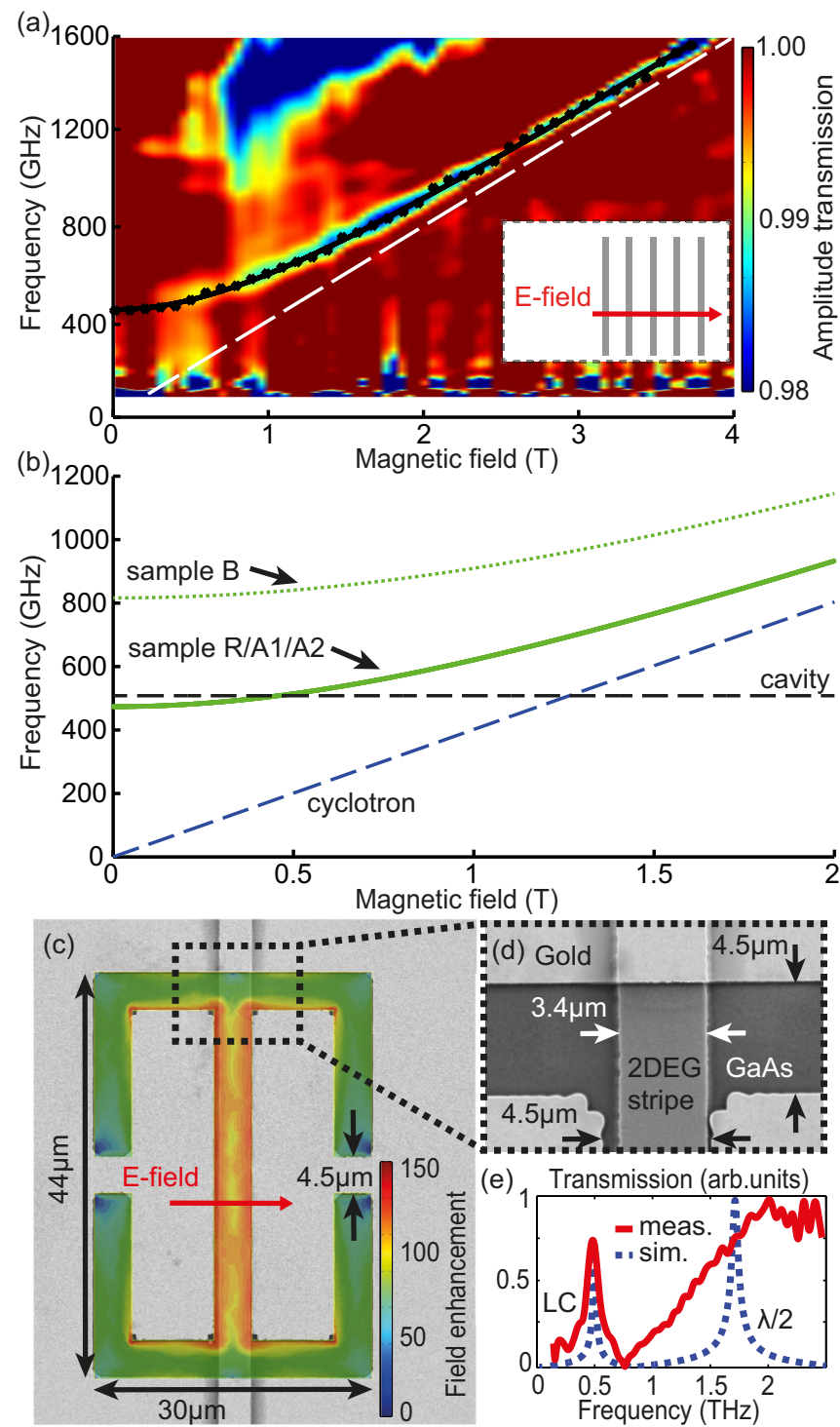

FIG. 1. (a) Bare MP resonance. Measured amplitude transmission vs $B$ field of sample R at $3 \mathrm{~K}$ (black dots) and fit (black line) using Eq. (2) (slow drifts of the background are subtracted with a linear fit). (Inset) Sample sketch and transmitted light polarization. (b) Bare cyclotron dispersion $\omega_{c}$ (blue dashed line), bare cavity frequency $\omega_{\text {cav }}$ (black dashed line), and the computed lowest-order MP dispersion expected for samples R/A1/A2 (green line) and sample B (green dashed line) are also shown. (c) SEM picture of A1 with a FE simulation of the in-plane electric-field distribution normalized to the incident field overlapped as a color map. The probing $\mathrm{THz}$ field is polarized across the gap. (d) Magnified SEM picture showing the stripe and the capacitive gap of the resonator and their alignment. Sample A2 is nominally identical apart from an additional 2-nm gate, while sample B has a 1.4- $\mu \mathrm{m}$-wide stripe instead. (e) Simulated and measured transmission spectrum of the bare resonator with light polarized as sketched in (c) shows the LC mode at $500 \mathrm{GHz}$ and $\lambda / 2$ resonance at $1.7 \mathrm{THz}$. The latter is far away from $\omega_{p}$ and thus negligible in our study.

width of $3.0 \mu \mathrm{m}$ is used due to depletion at the edges of the stripe [33]. Due to the small dimension, retardation effects are not relevant here [34] and no corrections to the simple 


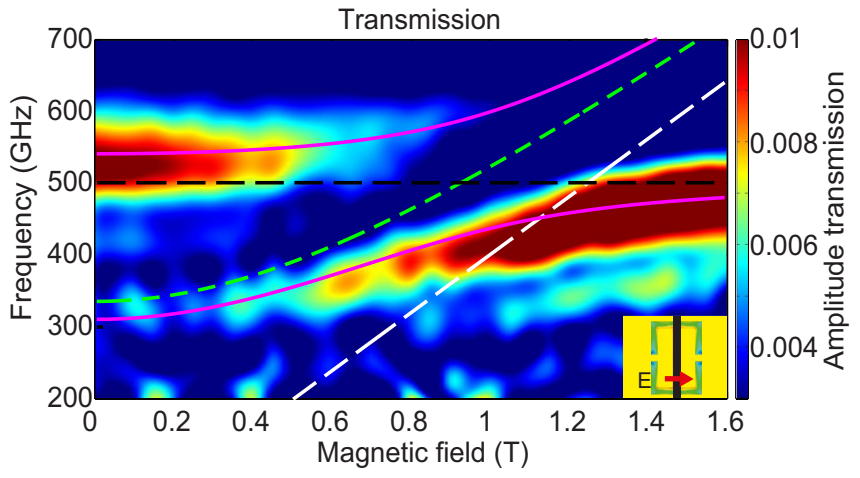

FIG. 2. Sample A1 (without gate). THz transmission spectra measured with THz-TDS at $3 \mathrm{~K}$ at different $B$ fields. The cavity frequency obtained from the high $B$-field limit (dashed black line) is $500 \mathrm{GHz}$. The dashed green line shows the bare MP dispersion inferred from fitting the magenta MPP curves (see Sec. IV). The effective mass is $0.070 \times m_{0}$. This defines the cyclotron dispersion (dashed white line). (Bottom right inset) Sample sketch and incident light polarization.

model in Eq. (2) are necessary. For the effective permittivity $\epsilon$ of the surrounding medium, the average permittivity of GaAs and vacuum is used; thus, $\epsilon=(12.89+1) / 2$. From the fit one then obtains an effective electron mass of $0.070 \times m_{0}$ and an electron density of $n_{s}=2.7 \times 10^{11} \mathrm{~cm}^{-2}$, corresponding to a plasmon frequency of $470 \mathrm{GHz}$. The density is consistent with transport measurements, and the mass is the same as the one obtained for the bare cyclotron dispersion measured in transmission. For clarity, Fig. 1(b) shows again the computed cyclotron dispersion (blue dashed line), the cavity frequency (black dashed line), and the MP dispersions for samples R, A1, A2 (green line), as well as for the narrower stripes of sample $\mathrm{B}$ (green dashed line).

The color map in Fig. 2 shows the $\mathrm{THz}$ transmission amplitude spectra of sample A1 at $3 \mathrm{~K}$ for different $B$ fields from 0 to $1.6 \mathrm{~T}$. As discussed before, the polariton branches result in transmission peaks since we are using complementary resonators [11]. A higher transmission amplitude indicates a more cavitylike polariton. One can see the upper polariton branch emerge from a horizontal line (shifted cavity frequency) at zero $B$ field and converge to the MP dispersion (matter part), similarly to cyclotron polaritons (CPs) [8]. In contrast, the lower polariton shows a fundamentally different behavior than CPs. It does not emerge from the linear cyclotron dispersion (dashed white line in Fig. 2) and converge to the empty cavity resonance frequency (black dashed line). Instead, the lower polariton already exists at zero $B$ field with a frequency of around $320 \mathrm{GHz}$. With increasing $B$ field it then crosses the cyclotron dispersion to then converge to the cavity frequency at high $B$ fields. It is clear that the bare MP dispersion takes over the role of the linear cyclotron dispersion as the nonradiative matter part. Despite the significant detuning of the MP and the cavity, the existence of two polaritons at zero $B$ field suggests that the ultrastrong coupling regime is already reached at zero $B$ field.

The two magenta curves in Fig. 2 also show the computed magnetoplasmon polariton (MPP) frequencies obtained from the model discussed in Sec. IV. Further, the bare MP dispersion
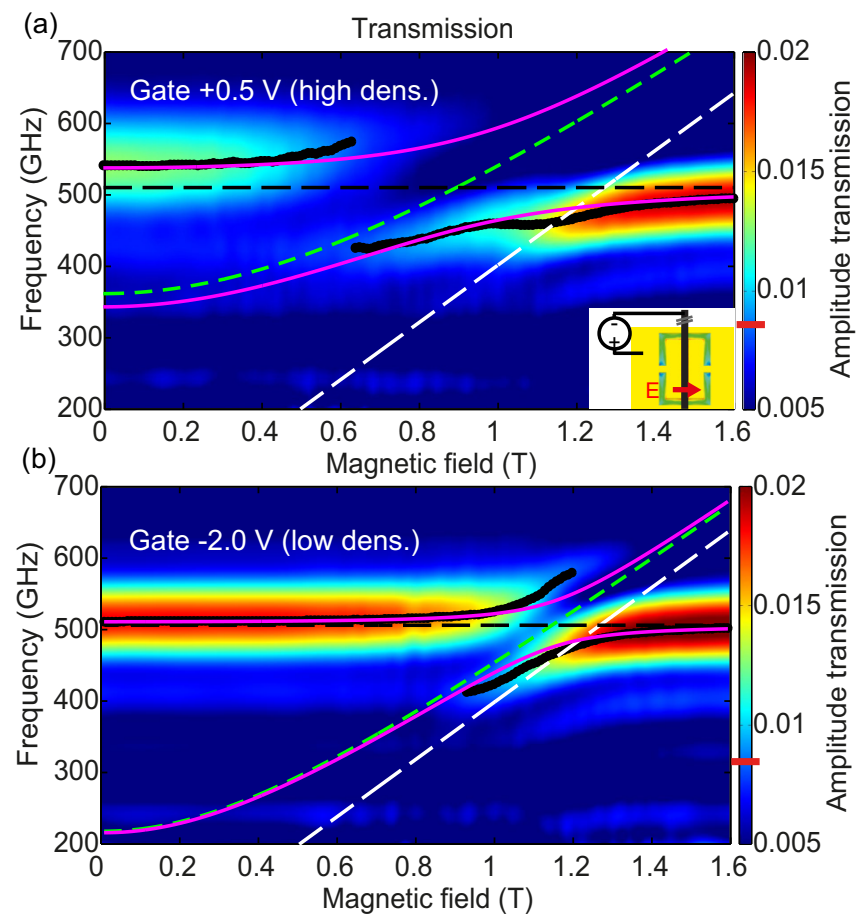

FIG. 3. Sample A2 (with chromium gate). THz amplitude transmission spectra (a) at $+0.5 \mathrm{~V}$ and (b) at $-2 \mathrm{~V}$ gate bias measured with THz-TDS as function of the $B$ field. Black dots mark local maxima above the threshold signal shown on the color bar (red ticks). The cavity frequency obtained from the high $B$-field limit (dashed black lines) is $506 \mathrm{GHz}$ for the A1 sample and $508 \mathrm{GHz}$ for the A2 sample. The dashed green lines show the bare MP dispersion inferred from fitting the magenta MPP curves (see Sec. IV). The plasmon frequencies at $B=0$ are 360 and $220 \mathrm{GHz}$ for (a) and (b), respectively. The effective mass is $0.070 \times m_{0}$. This defines the cyclotron dispersion (dashed white lines), which causes a deviation from the computed MPP curve when it crosses the lower polariton. (Bottom right inset) Sketch of sample with gate bias applied between resonator plane and 2DEG stripe and incident light polarization.

consistent with the polariton dispersion is shown in green. The free parameters in the model along with their value found by fitting the curve are the plasmon frequency $\omega_{p}=340 \mathrm{GHz}$ and the coupling $\Omega / \omega_{\text {cav }}=16 \%$. The effective mass found is identical with the value of $m^{*}=0.070 \times m_{0}$ found in the reference measurement in Fig. 1(a) and to the mass of the bare cyclotron dispersion. As expected, the light-matter coupling is lower than for resonators on a full $2 \mathrm{DEG}$, since the stripe fills a smaller fraction of the cavity volume compared to a full 2DEG [see Fig. 1(a)]. The obtained plasmon frequency in contrast is $130 \mathrm{GHz}$ lower than the $470 \mathrm{GHz}$ value obtained from transmission through uncovered 2DEG stripes of the same width [Fig. 1(a)]. This can be explained with an increased effective permittivity $\epsilon$ due to the presence of the metal close to the MP wave function. A FE simulation using CST microwave studio of a metal sheet near a 2DEG stripe confirms such a red shift (see Fig. 6 and Sec. IV for a detailed discussion).

Figures 3(a) and 3(b) show sample A2, which is a redo of A1 but with a 2 -nm-thick chromium gate on top of the resonators. The measurement with a gate bias of $+0.5 \mathrm{~V}$ to obtain a higher density is shown in Fig. 3(a). As expected, we find a 
higher plasmon frequency of $360 \mathrm{GHz}$ [see Eq. (2)]. Despite the higher density, the normalized light-matter coupling of $12 \%$ is lower than for sample A1, and the lower polariton is consequently not visible all the way to zero $B$ field anymore. The reason for the reduction is not fully clear but might be due to a less optimal resonator-stripe alignment.

Figure 3(b) shows again sample A2 at a gate bias of $-2 \mathrm{~V}$. The negative gate bias reduces the electron density and thus also the plasmon frequency from $360 \mathrm{GHz}$ down to $220 \mathrm{GHz}$ [see Eq. (2)]. One can observe a few clear differences to the measurement in Fig. 3(a), which we attribute to the nature of MPPs. First, the anticrossing at low electron density moves from $0.9 \mathrm{~T}$ at $+0.5 \mathrm{~V}$ gate voltage to $1.15 \mathrm{~T}$. For CPs no shift occurs for a change in carrier density since the matter part is the density-independent cyclotron dispersion.

Second, for the low-density measurement in Fig. 3(b) the transmission amplitude of the upper branch at $B=0 \mathrm{~T}$ has almost the same amplitude as at high $B$ fields. In contrast, the amplitude is significantly smaller at high carrier densities in Fig. 3(a). The latter is attributed to the higher plasmon frequency, which can push the system into the ultrastrong coupling regime already at $B=0 \mathrm{~T}$. This results in an uper polariton at $B=0 \mathrm{~T}$ that is less cavitylike and more weakly coupled to free space.

Further, the separation between the upper polariton and the bare cavity frequency of $30 \mathrm{GHz}$ is about 2 times larger than what is expected from CPs with the same coupling of $12 \%$ [35]. This also confirms that we are coupling to a MP and not to the cyclotron.

Third, the coupling reduces from $\Omega / \omega_{\text {cav }}=12 \%$ to around $6.5 \%$. If we assume that the coupling for MPPs also scales with the square root of the carrier density, the above tuning is roughly consistent with the tuning range of the plasmon frequency [which has the same carrier density dependence; see Eq. (2)].

It is further interesting to note that the strong gate dependence of the transmission allows to design an electrically tunable transmission device. In our sample, the optimal configuration is found at around $0.8 \mathrm{~T}$ [compare Figs. 3(a) and 3(b)], where the transmission of $520 \mathrm{GHz}$ can be reduced by a factor 5 by changing the gate from -2 to $+0.5 \mathrm{~V}$. Optimizing this system (by increasing the plasmon frequency) might make it possible to obtain such a switching behavior also at $0 \mathrm{~T}$ and with a higher extinction ratio.

Figure 4 shows a measurement of sample B that also has a $500-\mathrm{GHz}$ resonator but with a narrower stripe of $1.4 \mu \mathrm{m}$ and an effective width of $1 \mu \mathrm{m}$ passing through its gap. This shifts the MP frequency $\sqrt{3}$ times higher than for samples A1 and A2 to above $800 \mathrm{GHz}$. The presence of the metal probably does not reduce the frequency as much as in samples A1/A2, since the resonator gap is significantly wider than the 2DEG stripe, leaving a $2-\mu \mathrm{m}$ gap (compared to $<0.5 \mu \mathrm{m}$ ) between the metal and the 2DEG. In this configuration, we expect little to no coupling between the resonator and the plasmon due to the significant detuning. As shown in Fig. 4, we can still observe a clear coupling at $1.2 \mathrm{~T}$, between the resonator and an excitation in the 2DEG, which is consistent with the bare cyclotron dispersion with a slightly lighter effective mass of $m^{*}=0.066 \times m_{0}$. The top right inset shows the peak transmission amplitude versus $B$ field and an arrow marks where the cyclotron dispersion is

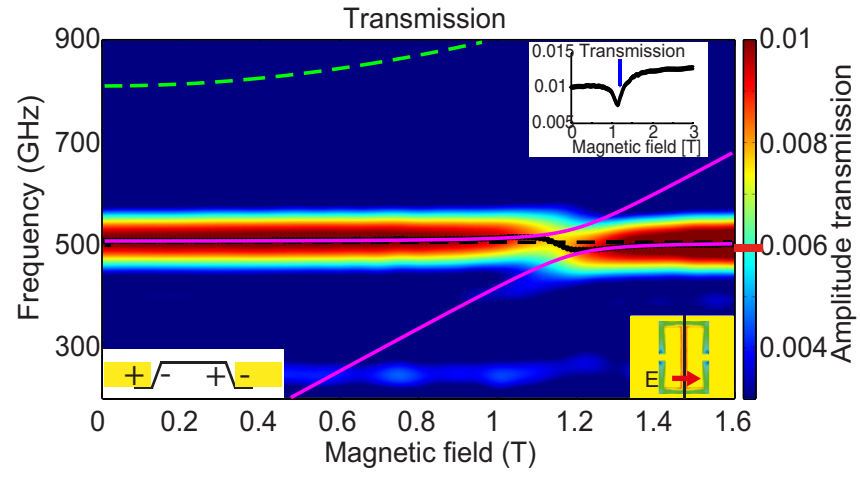

FIG. 4. Sample B. THz amplitude transmission spectra (not gated) measured with THz-TDS as function of the $B$ field. Black dots mark local maxima above the threshold signal marked on the color bar (red tick). The cavity frequency obtained from the high $B$-field limit (dashed black line) is $506 \mathrm{GHz}$. The dashed green line shows the estimated bare MP dispersion. It is expected to be at least $\sqrt{3}$ times higher than for samples $\mathrm{A} 1$ and $\mathrm{A} 2$ due to its 3-times-narrower width [see Eq. (2)] and a smaller effective $\epsilon$. The measurement data is best described by CPs (magenta lines), resulting from an anticrossing of the bare cyclotron dispersion with a lower effective mass of $0.066 \times m_{0}$ and the cavity resonance (dashed black line). (Bottom left inset) Sketch of sample cross section showing charge accumulation at the edges of the 2DEG stripe in response to the ac field applied by the resonator. (Bottom right inset) Sample sketch and incident light polarization. (Top right inset) Peak transmission as function of the $B$ field showing a dip at anticrossing.

resonant to the cavity. Figure 4 also shows the computed $\mathrm{CP}$ dispersion (magenta line) that is the result of the anticrossing between the linear cyclotron dispersion (white dashed line) and the cavity (black dashed line). The coupling strength estimated from the fit is $\Omega / \omega \approx 5 \%$. This is around 1 order of magnitude lower than the cyclotron coupling obtained between a resonator and full 2DEG, but relatively similar to the results obtained for the case $\omega_{p} \approx \omega_{\text {cav }}$ (samples A1/A2), if one considers the number of electrons in the cavity is 3 times lower, reducing the coupling by a factor $\sqrt{3}$. This is a surprising result, since the electron gas should be able to screen part of the external electric field by accumulating charge at the edges of the stripe (see Fig. 4, bottom left sketch)

It is interesting to compare our result to the one of Hatke et al. [36]. They also find two coexisting effective masses in wide electron gas stripes by measuring the longitudinal magnetoresistivity as function of $B$ field under microwave irradiation at and around the plasmon frequency. The first mass stems from the MP resonance causing a resistance peak due to heating of the electron gas. It is very close to the bulk GaAs mass. A $12 \%$ lower effective electron mass is determined by the so-called microwave-induced resistance oscillations (MIRO). They are a result of electron transitions between different Landau levels at the Fermi level and are therefore controlled by the cyclotron dispersion. Notably, this remains true above, at, and also below the plasmon frequency defined by the width of the stripe (e.g., see Fig. 1(b) in [36]). Further, unlike in the case of Shubnikov-de Haas oscillations, the position of those oscillations gives direct access to the effective mass of the underlying cyclotron dispersion. 


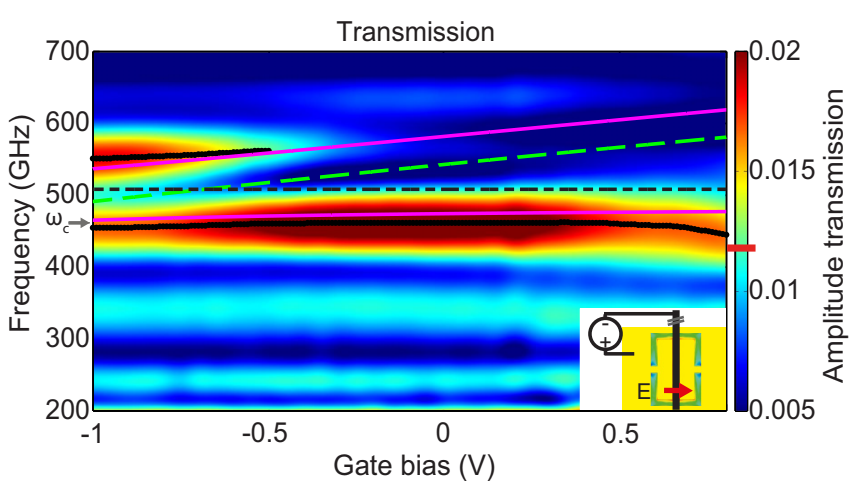

FIG. 5. Sample A2, gate sweep. THz amplitude transmission spectra measured as function of gate bias with THz-TDS at a fixed $B=1.1 \mathrm{~T}$. Black dots mark local maxima above the threshold signal marked on the color bar (red tick). The computed MPPs (magenta lines), the bare cavity frequency (dashed black line), and bare MP dispersion (dashed green line) are also shown, with the parameters obtained from the measurement in Fig. 3(b). We only assume the density is constant below $-1 \mathrm{~V}$ and linearly increases between -1 and $+0.8 \mathrm{~V}$. One can see the intensity and frequency increasing in the low-density side (left) of the plot, consistent with the expected behavior of MPPs (magenta lines). On the high-density (right) side of the plot the behavior is reversed, which is caused by the cyclotron coupling that gives a significant contribution due to growing detuning of the MP from the cavity. (Bottom right inset) Sketch of sample with gate bias applied between resonator plane and 2DEG stripe and incident light polarization.

In summary, the presence of the plasmon resonance introduced by etching a 2DEG stripe results in three distinct regimes. For $\omega_{p} \ll \omega_{\text {cav }}$, we obtain CPs as a result of the anticrossing of the cavity resonance with the cyclotron dispersion [8,11]. This is the regime, reached for infinite 2DEGs, where the plasmon frequency goes to zero. The second regime, $\omega_{p} \approx \omega_{\text {cav }}$, results in MPPs as shown in Fig. 3. The third regime $\omega_{p} \gg \omega_{\text {cav }}$ recovers CPs, but now with a significant screening and a lower effective mass, probably due to the presence of strong electron-electron interaction [36].

\section{Coexistence of MP and cyclotron}

The lower polariton branch in Fig. 3(a) clearly differs from our model (magenta line) between 1 and 1.2 T. This is the $B$ field range at which the cyclotron dispersion (white dashed line) is within the linewidth of the lower MPP branch. A similar but slightly less clear deviation is also observable in Figs. 2 and 3(b). This is further consistent with the result obtained from sample B, where we saw strong coupling to the cyclotron transition below the usually dominating plasmon resonance.

The coexistence of the two excitations is further confirmed by measuring transmission spectra at different gate voltages between -1 and $+0.8 \mathrm{~V}$ while keeping the $B$ field fixed at $1.1 \mathrm{~T}$. The result is shown in Fig. 5. The computed MP frequency at $1.1 \mathrm{~T}$ is shown as a green dashed curve. It is obtained assuming that the density changes linearly with the gate voltage between -1 and $+0.5 \mathrm{~V}$ and stays constant below $-1 \mathrm{~V}$. Below $-1 \mathrm{~V}$, no influence is observed on the transmission spectra, confirmed by all eight gate sweeps performed at different $B$ fields (not shown). We use the extracted effective plasmon frequencies from Fig. 3 and the formula 2 to obtain the green dashed line. The computed cyclotron frequency at $1.1 \mathrm{~T}$ is $460 \mathrm{GHz}$ and marked with a gray arrow in Fig. 5. Further, the computed MPP dispersions are shown (magenta lines), obtained with our model and assuming that the coupling scales with the square root of the carrier density, as it does for CPs [11]. Consistent with the MPP model, the upper MPP polariton branch increases in frequency as the density grows towards larger gate biases. The transmission at the upper polariton frequency decreases since the polariton becomes less cavitylike and more weakly coupled to free space. The lower polariton deviates from the MPP model as seen before in Figs. 3(a) and 3(b). The striking feature is that the transmission and amplitude of the lower polariton branch increases at first with increasing density (thus becoming more cavitylike, as expected), but then reduces again (to become less cavitylike again). We attribute this to the coexistence of the MP and cyclotron excitations in the system. At low densities, the MP is approximately resonant to the cavity. With increasing density (and thus increasing detuning between MP and resonator) the lower polariton becomes more cavitylike and therefore allows more transmission. At high densities, the cyclotron resonance coupling starts to kick in as its coupling becomes stronger and the previously dominant contribution from the MP starts to weaken due to detuning from the resonator. The latter also causes our MPP model to fail at high densities.

\section{THEORETICAL MODEL AND FE SIMULATION}

Here we present a theoretical description of the MPP dispersion based on an adapted theory by Hagenmüller et al. [37]. Their theory successfully predicts the coupling between a cyclotron transition in a two-dimensional electron gas under a strong $B$ field and a $\lambda / 2$ cavity resonator. This model is also applicable for an LC cavity on a 2DEG. See [11] for a detailed discussion.

The Landau gauge is used and a weakly excited electron gas is assumed, resulting in an approximately bosonic bright mode creation operator $b_{q_{x}}^{\dagger}$ associated with the cyclotron transition. The quantum number $q_{x}$ represents the relevant in-plane momentum of the cavity excitation $\mathbf{a}_{q_{x}}^{\dagger}$ with the energy $\hbar \omega_{q_{x}}$.

With a generalized Hopfield transformation, the Hamiltonian can be diagonalized. In the basis $\left(\mathbf{a}_{q_{x}}, b_{q_{x}}, \mathbf{a}_{q_{x}}^{\dagger}, b_{q_{x}}^{\dagger}\right)$, the eigenvalue problem takes the form

$$
M_{q_{x}} \mathbf{v}_{q_{x}}^{(i)}=\hbar \omega_{q_{x}}^{(i)} \mathbf{v}_{q_{x}}^{(i)},
$$

where $\hbar \omega_{q_{x}}^{(i)}$ are the magnetopolariton energies and $\mathbf{v}_{q_{x}}^{(i)}$ the corresponding eigenvectors with the index $i$ running over all polariton branches.

The infinite Hopfield matrix is then given by

$$
M_{q_{x}}=\left(\begin{array}{cccc}
\boldsymbol{\omega}_{q_{x}}+2 \boldsymbol{D}_{q_{x}} & i \boldsymbol{\Omega}_{q_{x}} & -2 \boldsymbol{D}_{q_{x}} & i \boldsymbol{\Omega}_{q_{x}} \\
-i \boldsymbol{\Omega}_{q_{x}}^{T} & \boldsymbol{\omega}_{0} & i \boldsymbol{\Omega}_{q_{x}}^{T} & 0 \\
2 \boldsymbol{D}_{q_{x}} & i \boldsymbol{\Omega}_{q_{x}} & -\boldsymbol{\omega}_{q_{x}}-2 \boldsymbol{D}_{q_{x}} & i \boldsymbol{\Omega}_{q_{x}} \\
i \boldsymbol{\Omega}_{q_{x}}^{T} & 0 & -i \boldsymbol{\Omega}_{q_{x}}^{T} & -\boldsymbol{\omega}_{0}
\end{array}\right) \text {, }
$$




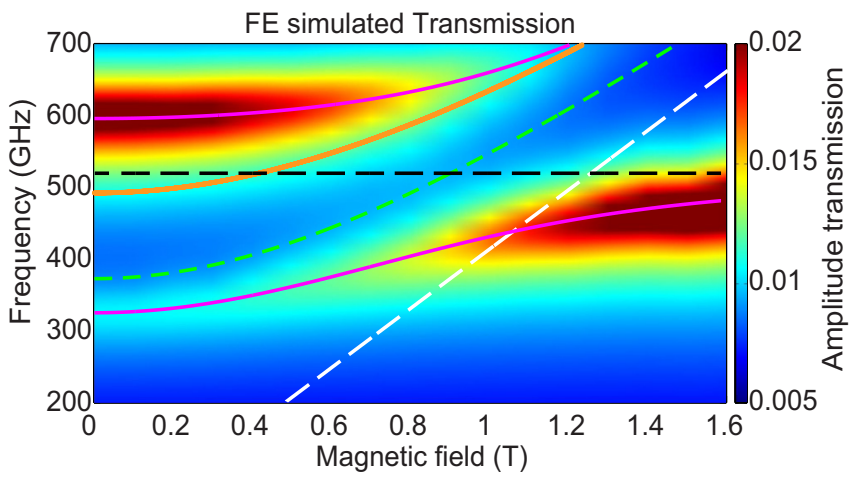

FIG. 6. FE simulation and theoretical model comparison. The color map shows the FE simulation of an electron gas stripe in the slit of the LC resonator. The FE simulated and then fitted bare cyclotron dispersion (white dashed line), cavity resonance frequency (black dashed line), and MP dispersion of the 2DEG stripe alone (orange line) are overlapped. The fitted analytic MPP dispersions from Eq. (6) are shown in magenta (see text), along with the inferred effective MP dispersion (green dashed line).

where the vector $\boldsymbol{\Omega}_{q_{x}}$ is the collective vacuum Rabi frequency and the matrix $\mathbf{D}_{q_{x}}=\boldsymbol{\Omega}_{q_{x}} \boldsymbol{\Omega}_{q_{x}}^{T} \omega_{0}^{-1}$ describes the self-interaction coming from the squared vector potential $\mathbf{A}_{e m}^{2}$.

In Hagenmüller et al. [37], $\omega_{0}$ describes the cyclotron transition frequency. We now alter the model by replacing $\omega_{c}$ with the MP frequency $\omega_{M P}$ from Eq. (2). The single-particle cyclotron and the collective MP excitation are quite different in nature, but both behave approximately bosonic.

Now, we assume only one cavity mode $\left(\omega_{q_{x}=0}=\omega_{\text {cav }}\right)$, an acceptable assumption in our situation with a coupling below $20 \%$. The relevant positive eigenvalues of the eigenproblem are then given by

$$
\omega_{(L P)}^{(U P)}=\frac{1}{\sqrt{2}} \sqrt{\omega_{M P}^{2}+4 \Omega^{2}+\omega_{\mathrm{cav}}^{2} \pm G},
$$

where

$$
G=\sqrt{-4 \omega_{M P}^{2} \omega_{\mathrm{cav}}^{2}+\left(-\omega_{M P}^{2}-4 \Omega^{2}-\omega_{\mathrm{cav}}^{2}\right)^{2}}
$$

describes the polariton gap. The smallest separation of the two branches $\omega^{(U P)}-\omega_{(L P)}=2 \Omega$ is reached when the MP dispersion becomes resonant with the cavity; thus, $\omega_{M P}=$ $\omega_{\text {cav }}=\omega$. To fit our measured MPP branches, we can now use Eq. (6), with the Rabi frequency $\Omega$ and $\omega_{p}$ as main free parameters. The latter comes from plugging Eq. (2) into (6). The effective mass therein is fixed from the measurement of the bare MP dispersion shown in Fig. 1(a). The cavity frequency is slightly adjusted $(<1 \%)$ to account for small differences in the processes of the three samples A1, A2, and B.

Figure 6 shows an FE simulation of sample A1 using again CST microwave studio. The simulated field distribution and transmission of the bare cavity are shown in Figs. 1(b) and 1(c), respectively. The bare 2DEG stripe is modeled as a electric gyrotropic medium. The thickness of the stripe in the $z$ direction is chosen to be $250 \mathrm{~nm}$, to improve the numerical stability. The plasma frequency containing the electron density and the collision frequency describing the damping are chosen to reproduce the measured bare MP dispersion shown in Fig. 1(a). The cavity made out of gold is simulated using the standard gold lossy metal from the CST material library.

The FE simulated MP dispersion of the 2DEG stripe alone is fitted with the model in Eq. (2) and shown as an orange curve in Fig. 6. With the model parameters used, we obtain a plasmon frequency of $492 \mathrm{GHz}$. The FE simulation of the combined resonator-stripe system is shown as a color map in the latter figure. For comparison, the MPP dispersions (magenta) fitted using the analytic Eq. (6) are superimposed. The free parameters along with their values are the normalized light-matter coupling $\Omega /\left.\omega_{\text {cav }}\right|_{\text {sim }}=22 \%$ and the bare plasmon frequency $\omega_{p}=372 \mathrm{GHz}$. The corresponding bare MP dispersion is shown as a dashed green line. The cyclotron dispersion corresponding to the effective mass of $m^{*}=0.070 \times m_{0}$ is also shown as a dashed white line.

As also shown in Fig. 6, we obtain significantly different results for the FE simulated plasmon frequency of the 2DEG stripe alone (orange line) and the plasmon frequency inferred from the analytic MPP dispersions from Eq. (6) (green dashed line). The difference of $120 \mathrm{GHz}$ at zero $B$ field stems from the presence of metal right next to the electron gas in the second case, increasing the effective electric permittivity the plasmon sees. This is consistent with the measurements where we saw a shift or around $130 \mathrm{GHz}$ (see Fig. 2 and text).

\section{CONCLUSION AND OUTLOOK}

In conclusion, we present a cavity quantum electrodynamic system, which shows ultrastrong coupling to magnetoplasmons in an etched 2DEG stripe. Thanks to the special geometry, we can spectroscopically study the cyclotron resonance in the presence of the strong magnetoplasmon resonance. This system has three regimes. In the first, where $\omega_{p} \ll \omega_{\text {cav }}$, normalized light-matter coupling ratios of around $100 \%$ have been demonstrated with cyclotron polaritons [11]. Second, with $\omega_{p} \approx \omega_{\mathrm{cav}}$, we obtain MPPs with a coupling of around $10 \%-20 \%$. It can further result in ultrastrong coupling at zero $B$ field. An adopted model from Hagenmüller et al. [37] can successfully describe their dispersion. A good agreement with finite element simulations is also obtained. We can further spectroscopically observe the coexistence of the bare cyclotron resonance in this regime. Third, in the regime $\omega_{p} \gg \omega_{\text {cav }}$ we observe again a coupling to the cyclotron excitation, but this time associated with a lower effective mass of $0.066 \times m_{0}$.

As an outlook, the present system can be readily expanded to a Hall bar completely inside the slit of the LC resonator. This would make it possible to study quantum transport in the presence of ultrastrong coupling. Since both electronic transport and the ultrastrong coupling to cyclotron polaritons involve electrons at the Fermi surface, we expect significant effects in the longitudinal and also transversal resistance curves. As illustrated by the MIRO effect (see [38] for a review), microwaves have a significant impact on the transport in high-mobility 2DEGs. It is thus conceivable 
that vacuum fluctuations at the same frequency might cause MIRO-like oscillations as well. Even increases in the mobility might be possible, since the cavity part of the polariton can provide a long-range coherence that allows the electron wave function to avoid defects in the Hall bar. This was previously shown experimentally [39] and theoretically [40,41] for exciton transport in highly disordered molecular systems.

\section{ACKNOWLEDGMENTS}

The authors acknowledge financial support from the ERC Advanced grant Quantum Metamaterials in the Ultra Strong Coupling Regime (MUSiC) with the ERC Grant No. 340975. The authors also acknowledge financial support from the Swiss National Science Foundation (SNF) through the National Centre of Competence in Research Quantum Science and Technology (NCCR QSIT).
[1] C. Ciuti, G. Bastard, and I. Carusotto, Phys. Rev. B 72, 115303 (2005).

[2] A. A. Anappara, S. De Liberato, A. Tredicucci, C. Ciuti, G. Biasiol, L. Sorba, and F. Beltram, Phys. Rev. B 79, 201303 (2009).

[3] Y. Todorov, A. M. Andrews, I. Sagnes, R. Colombelli, P. Klang, G. Strasser, and C. Sirtori, Phys. Rev. Lett. 102, 186402 (2009).

[4] T. Niemczyk, F. Deppe, H. Huebl, E. Menzel, F. Hocke, M. Schwarz, J. J. García-Ripoll, D. Zueco, T. Hümmer, E. Solano et al., Nat. Phys. 6, 772 (2010).

[5] P. Forn-Díaz, J. Lisenfeld, D. Marcos, J. J. García-Ripoll, E. Solano, C. J. P. M. Harmans, and J. E. Mooij, Phys. Rev. Lett. 105, 237001 (2010).

[6] V. M. Muravev, I. V. Andreev, I. V. Kukushkin, S. Schmult, and W. Dietsche, Phys. Rev. B 83, 075309 (2011).

[7] M. Geiser, F. Castellano, G. Scalari, M. Beck, L. Nevou, and J. Faist, Phys. Rev. Lett. 108, 106402 (2012).

[8] G. Scalari, C. Maissen, D. Turčinková, D. Hagenmüller, S. De Liberato, C. Ciuti, C. Reichl, D. Schuh, W. Wegscheider, M. Beck et al., Science 335, 1323 (2012).

[9] V. M. Muravev, P. A. Gusikhin, I. V. Andreev, and I. V. Kukushkin, Phys. Rev. B 87, 045307 (2013).

[10] Q. Zhang, M. Lou, X. Li, J. L. Reno, W. Pan, J. D. Watson, M. J. Manfra, and J. Kono, Nat. Phys. 12, 1005 (2016).

[11] C. Maissen, G. Scalari, F. Valmorra, M. Beck, J. Faist, S. Cibella, R. Leoni, C. Reichl, C. Charpentier, and W. Wegscheider, Phys. Rev. B 90, 205309 (2014).

[12] F. Stern, Phys. Rev. Lett. 18, 546 (1967).

[13] C. Grimes and G. Adams, Phys. Rev. Lett. 36, 145 (1976).

[14] S. J. Allen Jr, D. C. Tsui, and R. A. Logan, Phys. Rev. Lett. 38, 980 (1977).

[15] T. Theis, J. Kotthaus, and P. Stiles, Solid State Commun. 26, 603 (1978).

[16] T. N. Theis, Surf. Sci. 98, 515 (1980).

[17] D. Heitmann, Surf. Sci. 170, 332 (1986).

[18] M. S. Kushwaha, Surf. Sci. Rep. 41, 1 (2001).

[19] J. Łusakowski, Semicond. Sci. Technol. 32, 013004 (2016).

[20] Z. Fei, A. S. Rodin, G. O. Andreev, W. Bao, A. S. McLeod, M. Wagner, L. M. Zhang, Z. Zhao, M. Thiemens, G. Dominguez et al., Nature 487, 82 (2012).

[21] L. Ju, B. Geng, J. Horng, C. Girit, M. Martin, Z. Hao, H. A. Bechtel, X. Liang, A. Zettl, Y. R. Shen et al., Nat. Nanotechnol. 6, 630 (2011).

[22] P. Q. Liu, F. Valmorra, C. Maissen, J. Keller, G. Scalari, and J. Faist, in SPIE OPTO (International Society for Optics and Photonics, Bellingham, WA, 2015), pp. 935707-935707.
[23] A. Muravjov, D. Veksler, V. Popov, O. Polischuk, N. Pala, X. Hu, R. Gaska, H. Saxena, R. Peale, and M. Shur, Appl. Phys. Lett. 96, 042105 (2010).

[24] V. M. Muravev, P. A. Gusikhin, I. V. Andreev, and I. V. Kukushkin, Phys. Rev. Lett. 114, 106805 (2015).

[25] P. Q. Liu, I. J. Luxmoore, S. A. Mikhailov, N. A. Savostianova, F. Valmorra, J. Faist, and G. R. Nash, Nat. Commun. 6, 8969 (2015).

[26] G. C. Dyer, G. R. Aizin, S. J. Allen, A. D. Grine, D. Bethke, J. L. Reno, and E. A. Shaner, Nat. Photonics 7, 925 (2013).

[27] M. Białek, M. Czapkiewicz, J. Wróbel, V. Umansky, and J. Łusakowski, Appl. Phys. Lett. 104, 263514 (2014).

[28] Y. Li, H. Yan, D. B. Farmer, X. Meng, W. Zhu, R. M. Osgood, T. F. Heinz, and P. Avouris, Nano Lett. 14, 1573 (2014).

[29] I. V. Kukushkin, V. M. Muravev, J. H. Smet, M. Hauser, W. Dietsche, and K. von Klitzing, Phys. Rev. B 73, 113310 (2006).

[30] O. M. Fedorych, M. Potemski, S. A. Studenikin, J. A. Gupta, Z. R. Wasilewski, and I. A. Dmitriev, Phys. Rev. B 81, 201302 (2010).

[31] V. M. Muravev, I. V. Andreev, S. I. Gubarev, V. N. Belyanin, and I. V. Kukushkin, Phys. Rev. B 93, 041110 (2016).

[32] J. Kröll, J. Darmo, and K. Unterrainer, Opt. Express 15, 6552 (2007).

[33] D. Smetnev, V. M. Muravev, I. Andreev, and I. V. Kukushkin, JETP Lett. 94, 137 (2011).

[34] S. A. Mikhailov and N. A. Savostianova, Phys. Rev. B 71, 035320 (2005).

[35] G. Scalari, C. Maissen, S. Cibella, R. Leoni, C. Reichl, W. Wegscheider, M. Beck, and J. Faist, Il Nuovo Saggiatore 31, 3 (2015).

[36] A. T. Hatke, M. A. Zudov, J. D. Watson, M. J. Manfra, L. N. Pfeiffer, and K. W. West, Phys. Rev. B 87, 161307(R) (2013).

[37] D. Hagenmüller, S. De Liberato, and C. Ciuti, Phys. Rev. B 81, 235303 (2010).

[38] I. Dmitriev, A. Mirlin, D. Polyakov, and M. Zudov, Rev. Mod. Phys. 84, 1709 (2012).

[39] E. Orgiu, J. George, J. Hutchison, E. Devaux, J. Dayen, B. Doudin, F. Stellacci, C. Genet, J. Schachenmayer, C. Genes et al., Nat. Mater. 14, 1123 (2015).

[40] J. Feist and F. J. Garcia-Vidal, Phys. Rev. Lett. 114, 196402 (2015).

[41] J. Schachenmayer, C. Genes, E. Tignone, and G. Pupillo, Phys. Rev. Lett. 114, 196403 (2015). 